Article

\title{
Antibacterial Activity of Phytochemicals Isolated from Atractylodes japonica against Methicillin-Resistant Staphylococcus aureus
}

Seung-Il Jeong ${ }^{1}$, Seon-Young Kim ${ }^{1}$, Sang-Jun Kim ${ }^{1}$, Byung-Soon Hwang ${ }^{1}$, Tae-Ho Kwon ${ }^{1}$, Kang-Yeol Yu ${ }^{1}$, Seung-Ho Hang ${ }^{2}$, Koji Suzuki ${ }^{3}$ and Kang-Ju Kim ${ }^{4, *}$

1 Jeonju Biomaterials Institute, Jeonju 561-360, Korea; E-Mails: seungil@paran.com (S.-I.J.); seon02@jbmi.re.kr (S.-Y.K.); process95@jbmi.re.kr (S.-J.K.); hbs2015@nate.com (B.-S.H.); thkwon@jbmi.re.kr (T.-H.K.);kangyu@jbmi.re.kr (K.-Y.Y.)

2 Department of Laboratory Medicine, Veterans Hospital, Daejeon, 306-830, Korea; E-Mail: microhemato@hanmail.net (S.-H.H.)

3 GL Sciences Inc, Tokyo, Japan; E-Mail: ksuzuki@gls.co.jp (K.S.)

4 Department of Oral Microbiology, School of Dentistry and Biotechnology Institute, Wonkwang University, Iksan 570-749, Korea

* Author to whom correspondence should be addressed; E-Mail: kjkimom@wonkwang.ac.kr (K.-J.K.); Tel.: +82-63-850-6858; Fax: +82-63-850-7313.

Received: 7 September 2010 / Accepted: 19 October 2010 / Published: 21 October 2010

\begin{abstract}
Methicillin-resistant Staphylococcus aureus (MRSA) has been emerging worldwide as one of the most important problems in communities and hospitals. Therefore, new agents are needed to treat acute oral infections from MRSA. In this study, antibacterial compounds from the roots of Atractylodes japonica (A. japonica) were isolated and characterized. The compounds were isolated from the root extracts using HPLC-piloted activity-guided fractionations. Four A. japonica compounds were isolated and identified as atractylenolide III (1), atractylenolide I (2), diacetylatractylodiol [(6E,12E)-tetradeca-6,12diene-8,10-diyne-1,3-diol diacetate, TDEYA, 3). and (6E,12E)-tetradecadiene-8,10-diyne1,3-diol (TDEA, 4), which was obtained by hydrolysis of TDEYA. The minimum inhibitory concentrations (MICs) was determined in the setting of clinical MRSA isolates. Compound 4 showed anti-MRSA activity with a MIC value of $4-32 \mu \mathrm{g} / \mathrm{mL}$. The overall results provide promising baseline information for the potential use of the extract of $A$. japonica as well as some of the isolated compounds in the treatment of bacterial infections.
\end{abstract}


Keywords: Atractylodes japonica; MRSA; MIC/MBC

\section{Introduction}

Methicillin-resistant Staphylococcus aureus (MRSA) accounts for a large proportion of hospitalacquired infections and is considered a serious problem because of its multi-drug resistant properties. Currently, vancomycin and its analog teicoplanin are the most effective antibiotics for MRSA infections, but their clinical use often results in unexpected side effects and the development of vancomycin-resistant $S$. aureus infections. The search for better drugs to combat MRSA infection is urgently needed.

Atractylodes japonica (Compositae) has traditionally been used for the treatment of water retention in the body. Administration of the aqueous extract of Atractylodes japonica to humans causes diuresis and its alcohol extract also shows a diuretic effect in mice [1,2]. Atractylodes japonica is known to be effective for the control of pain and treatment of arthritis. It was reported that the Atractylodes family possesses anti-inflammatory activity [3], and modulates the intestinal immune system [4]. Atractylon, a major component, and its derivatives isolated from the rhizome were shown to have antihepatic properties [5]. The sequiterpenoid diacetyl atractylodiol and its derivatives were isolated from the nonpolar fraction [2]. Recently, it was reported that A. japonica root extract protects osteoblastic MC3T3E1 cells against hydrogen peroxide-induced inhibition of osteoblastic differentiation [6]. However, the antibacterial effects of $A$. japonica on MRSA has not been evaluated. In the course of our ongoing project on the detection of bioactive compound from medicinal plants, the $\mathrm{CHCl}_{3}$-soluble extract of roots of $A$. japonica was found to exhibit distinctive antibacterial activity.

\section{Results and Discussion}

Today, with the emergence of antibiotic-resistant pathogens like MRSA, a new approach to natural products must be taken. These natural products are more and more in demand due to their benefits without side effects. Therefore, our ongoing efforts to find bioactive natural products have led us to study the antibacterial activity of $A$. japonica, which has been known to possess a variety of pharmacological properties against arthritis, bronchitis and respiratory infectious disease and to contain more than 50 phytochemicals, including atractylon and its derivatives [7,8], sesquiterpenoids [9] and diacetyl atractylodiol including its derivatives [10,11]. To elucidate the antibacterial effects of A. japonica, the methanol extracts of $A$. japonica rhizome were fractionized with $n$-hexane, $\mathrm{CHCl}_{3}$, EtOAc, and $n$-BuOH. The fractions were tested for MIC determination using the microdilution broth method. The results were recorded as MIC values in Table 1. The $\mathrm{CHCl}_{3}$ fraction of $A$. japonica roots showed good antibacterial effects against two strains of $S$. aureus. The $\mathrm{MIC}$ of $\mathrm{CHCl}_{3}$ fraction shows equal efficacy to ampicillin at $32 \mu \mathrm{g} / \mathrm{mL}$ against ATCC 33591. This is an encouraging result in regards to the ability of MRSA to be resistant to most antibiotics. By further purifying the fractions, compounds 1, 2 and 3 were isolated from the $\mathrm{CHCl}_{3}$ fraction and were confirmed to be atractylenolide III (1), atractylenolide I (2), and (6E,12E)-tetradeca-6,12-diene-8,10-diyne-1,3-diol diacetate (diacetylatractylodiol, TDEYA, 3), respectively, by the comparison of their spectral data with those in 
the references [12]. Also, (6E,12E)-tetradecadiene-8,10-diyne-1,3-diol (TDEA, 4) [11] was obtained by hydrolysis of TDEYA (3) (Figure 1).

Table 1. Antimicrobial activity of $A$. japonica root $\mathrm{MeOH}$ extract and $n$-hexane, $\mathrm{CHCl}_{3}$, EtOAc, and $n-\mathrm{BuOH}$ fractions against $S$. aureus (ATCC 33591, ATCC 25923) strains.

\begin{tabular}{lcccccc}
\hline \multirow{2}{*}{ S. aureus strain } & $\mathbf{M e O H}$ & Hexane & $\mathbf{C H C l}_{\mathbf{3}}$ & $\mathbf{E t O A c}$ & $\mathbf{B u O H}$ & Ampicillin \\
\cline { 2 - 7 } & 64 & 128 & 32 & 128 & 128 & 32 \\
\hline ATCC 33591 & 64 & 64 & 32 & 64 & 128 & 0.125 \\
ATCC 25923 & &
\end{tabular}

The isolated compounds after were tested against different strains of S. aureus as shown in Table 2 . Among the obtained isolates, TDEA (4) showed antibacterial activity with MICs ranging from 4 to $32 \mu \mathrm{g} / \mathrm{mL}$ independently.

Figure 1. Chemical structures of isolated compounds from roots of $A$. japonica: atractylenolide III (1); atractylenolide I (2); (6E, 12E)-tetradecadiene-8,10-diyne-1,3-diol diacetate (diacetylatractylodiol TDEYA, 3); (6E, 12E)-tetradecadiene-8,10-diyne-1,3-diol (TDEA, 4).

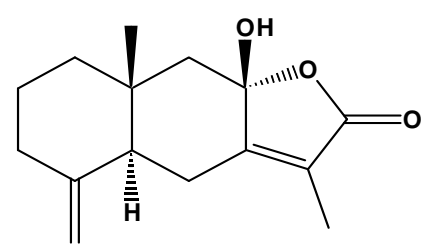

atractylenolide III (1)<smiles>C=C1CCC[C@]2(C)C=C3OC(=O)C(C)=C3C[C@]12C</smiles>

atractylenolide I (2)

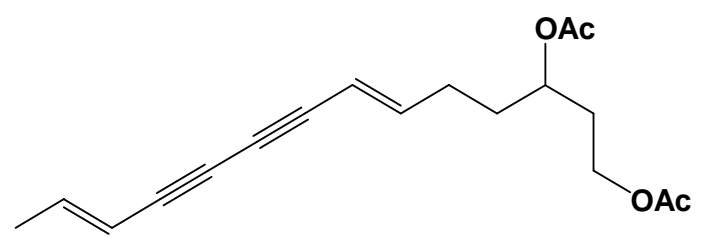

(6E, 12E)-tetradecadiene-8,10-diyne-1,3-diol diacetate (diacetylatractylodiol TDEYA, 3)<smiles>CC=CC#CC#CC=CCCC(O)CCO</smiles>

(6E, 12E)-teradecadiene-8,10-diyne-1,3-diol (TDEA, 4)

Also, we investigated phytochemical profiles in A. japonica after separation using the developed Smart HPLC method. Typical chromatograms of the $\mathrm{MeOH}$ extract of $A$. japonica roots are shown in 
Figure 2. The identification of investigated compounds was carried out by comparison of their retention time and UV spectra with those obtained injecting standards in the same conditions, or spiking the samples with stock standard solutions. The developed Smart LC method indicated that the principle contained in the $\mathrm{MeOH}$ extract of $A$. japonica contained atractylenolide III (1), atractylenolide I (2), and (6E,12E)-tetradeca-6,12-diene-8,10-diyne-1,3-diol diacetate (diacetylatractylodiol, TDEYA, 3), respectively. On Smart HPLC, (6E,12E)-tetradecadiene-8,10-diyne1,3-diol (TDEA) was the main active component in the tested $A$. japonica that contributed to the antibacterial effect of MRSA. TDEA(4) was obtained by hydrolysis of TDEYA, which was not detected on HPLC in A. japonica extract.

Additionally, among the five different fractions, the chloroform fraction of $A$. japonica demonstrated a higher inhibitory activity against MRSA due to its bioactive constituents, in agreement with our Smart HPLC study.

Figure 2. Smart HPLC chromatographic fingerprints of $\mathrm{MeOH}$ extract of A. japonica. The peaks marked with 2, 6 and 7 are atractylenolide III, atractylenolide I, (6E,12E)teradecadiene-8,10-diyne-1,3-diol diacetate (diacetylatractylodiol, TDEYA), respectively. The separation conditions are described in the Smart HPLC chromatographic conditions.

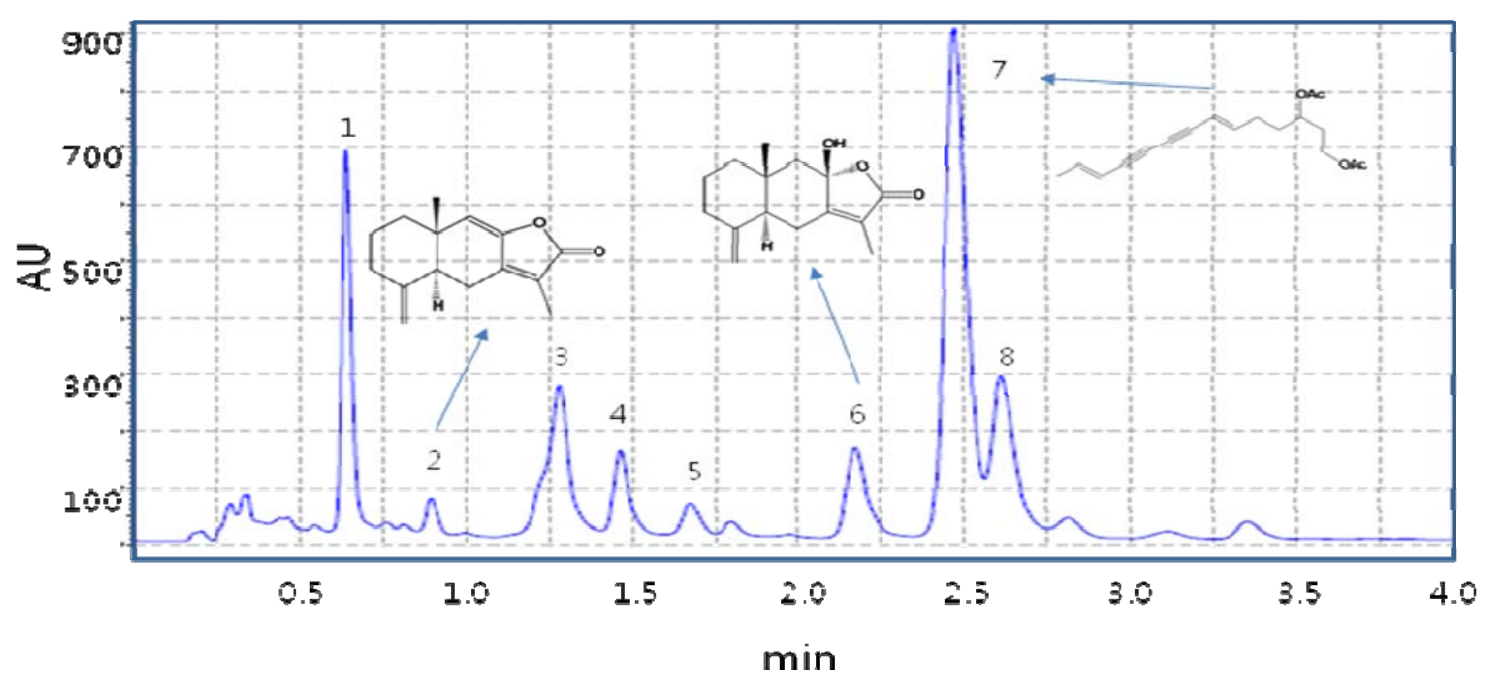

The MIC values of the isolated compounds against MRSA are summarized in Table 2. Compounds 1, 2, and 3 showed inhibitory effect against S. aureus ATCC33591 (standard MRSA), S. aureus ATCC25923 (standard MSSA) as well as clinical isolates of MRSA, with MIC values of 8 to 128,8 to 256 and 16 to $128 \mu \mathrm{g} / \mathrm{mL}$, respectively. Compound 4 showed a significant MIC value (4 to $32 \mu \mathrm{g} / \mathrm{mL}$ ) in comparison with the other compounds.

In conclusion, the isolated compounds are expected to be useful in the future for the study of antiMRSA agents. However, for medicinal purposes, the safety and toxicity of these compounds need to be addressed. Also, further study is needed using vancomycin as a positive control for MRSA. 
Table 2. The MICs/MBCs of $A$. japonica $\mathrm{CHCl}_{3}$ fraction (AJCH), atractylenolide III (1), atractylenolide I (2), (6E,12E)-tetradecadiene-8,10-diyne-1,3-diol diacetate (3) and $(6 E, 12 E)$-tetradecadiene-8,10-diyne-1,3-diol (4), and ampicillin (AM) against S. aureus strains.

\begin{tabular}{llcrrrrrr}
\hline & & & \multicolumn{7}{c}{ MIC $(\boldsymbol{\mu g} / \mathbf{m L})$} \\
\cline { 4 - 9 } S. aureus strain & Class & mecA gene & AJCH & $\mathbf{1}$ & $\mathbf{2}$ & $\mathbf{3}$ & $\mathbf{4}$ & AM \\
\hline ATCC 25923 & MSSA & - & 32 & 64 & 64 & 32 & 16 & 0.12 \\
ATCC 33591 & MRSA & + & 32 & 128 & 256 & 64 & 16 & 32 \\
Clinical isolates & & & & & & & & 25 \\
S. aureus 78 & MRSA & + & 64 & 64 & 64 & 64 & 8 & 256 \\
S. aureus M11 & MRSA & + & 64 & 64 & 64 & 16 & 8 & 256 \\
S. aureus 21-8 & MRSA & + & 64 & 32 & 32 & 128 & 4 & 256 \\
S. aureus 6-2 & MRSA & + & 128 & 64 & 64 & 64 & 8 & 256 \\
S. aureus 7-3 & MRSA & + & 64 & 64 & 64 & 64 & 32 & 256 \\
S. aureus 8-4 & MRSA & + & 64 & 128 & 128 & 64 & 16 & 256 \\
S. aureus 9-5 & MRSA & + & 256 & 128 & 128 & 32 & 16 & 256 \\
S. aureus 13-7 & MRSA & + & 32 & 32 & 32 & 32 & 4 & 128 \\
S. aureus 27-9 & MRSA & + & 64 & 32 & 128 & 64 & 8 & 128 \\
S. aureus 47-10 & MRSA & + & 32 & 32 & 32 & 64 & 16 & 512 \\
S. aureus 105-13 & MRSA & + & 32 & 8 & 8 & 64 & 4 & 256 \\
S. aureus 106-14 & MRSA & + & 32 & 16 & 16 & 32 & 4 & 128 \\
\hline
\end{tabular}

(+) positive, (-) negative

\section{Experimental}

\subsection{Plant material}

The fresh roots of Atractylodes japonica Koidz (Family Compositae) were collected in July 2009 at Jinan, Jeonbuk province, Korea. The plant was authenticated by Prof. Hong-Jun Kim, College of Oriental Medicine, Woosuk University. A voucher specimen (No. JSI 62) was deposited in the Herbarium of College of Oriental Medicine, Woosuk University.

\subsection{Extraction and isolation of test material}

Air-dried and powdered roots of A. japonica $(3.0 \mathrm{~kg})$ were extracted with two portions of $\mathrm{MeOH}$ $(10 \mathrm{~L})$ for one week at room temperature. To give $319 \mathrm{~g}$ of dark-brown extract, which was suspended in water. The suspension was extracted with hexane, $\mathrm{CHCl}_{3}$, EtOAc and $n-\mathrm{BuOH}$, respectively, and the organic layer was concentrated to dryness in vacuo to give the hexane (48 g), $\mathrm{CHCl}_{3}(62 \mathrm{~g})$, EtOAc $(23 \mathrm{~g})$, and $n-\mathrm{BuOH}$ fractions $(61 \mathrm{~g})$. The $\mathrm{CHCl}_{3}$ soluble portion $(42 \mathrm{~g})$ was chromatographed on silica gel $(6 \mathrm{~cm}$ i.d. $\times 50 \mathrm{~cm}, 1.5 \mathrm{~kg})$ eluted stepwise with a mixture of EtOAc and $n$-hexane $(16: 1 \rightarrow 8: 1 \rightarrow$ $4: 1 \rightarrow 2: 1 \rightarrow 0: 1$, each $800 \mathrm{~mL}$ ), yielding seven fractions (A1-A7). Fraction A1 was purified by prepLC JAI gel W252 column; 100\% MeOH; flow rate $3.5 \mathrm{~mL} / \mathrm{min}$; UV detection at $254 \mathrm{~nm}$ ) to give $\mathbf{1}$ (15 mg). Fraction A3 was also applied to a prep-LC (JAI gel W252 column; 100\% MeOH; flow rate $3.5 \mathrm{~mL} / \mathrm{min}$; UV detection at $254 \mathrm{~nm})$ to give $2(30 \mathrm{mg})$ and $3(12 \mathrm{mg})$. These compounds were identified as atractylenolide III (1), atractylenolide I (2), and (6E,12E)-tetradeca-6,12-diene-8,10diyne-1,3-diol diacetate (diacetyl-atractylodiol, TDEYA, 3) [12]. Compound 4 ((6E,12E)tetradecadiene-8,10-diyne-1,3-diol, TDEA) [11] was obtained by hydrolysis of $\mathbf{3}$ (see Figure 1). 


\subsection{Hydrolysis of TDEYA(3)}

A solution of $3(20 \mathrm{mg})$ in $3 \% \mathrm{~K}_{2} \mathrm{CO}_{3}$ (cat.)/MeOH $(7 \mathrm{~mL})$ was heated at $60{ }^{\circ} \mathrm{C}$ for $10 \mathrm{~min}$. After cooling, the solution was neutralized with $1 \mathrm{~N} \mathrm{HCl}$, and $\mathrm{MeOH}$ was extracted with $\mathrm{Et}_{2} \mathrm{O}$ and then washed with deionized water. The organic layer was dried over anhydrous $\mathrm{Na}_{2} \mathrm{SO}_{4}$ and evaporated in vacuo to obtain compound $\mathbf{4}$ as a pale yellow amorphous powder $(8 \mathrm{mg})$.

\subsection{Chromatographic fingerprint conditions}

A LC800 series (Smart LC, GL sciences, Tokyo, Japan) system equipped with binary solvent delivery pump, auto sampler, degasser, system oven and UV-visible detector was used to achieve Smart LC fingerprints. The chromatographic separation was carried out on a $2.1 \mathrm{~mm} \times 50 \mathrm{~mm}, 2 \mu \mathrm{m}$ particle, Innertsil ODS-4 C18 column (GL sciences, Japan) maintained at $40{ }^{\circ} \mathrm{C}$. The mobile phase consisting a mixture $0.05 \%$ aqueous phosphoric acid and acetonitrile in the ratio of 34:66 (v/v) with flow rate of $0.5 \mathrm{~mL} / \mathrm{min}$ was employed. The detector wavelength was monitored at $254 \mathrm{~nm}$. All injection volumes of sample and standards were $1.5 \mu \mathrm{L}$.

\subsection{Antibiotics}

All antibiotics, including ampicillin was purchased from Sigma Chemical Co. (St Louis, MO, USA).

\subsection{Bacterial strains}

The 12 MRSA isolates used in this study were clinical isolates from Wonkwang University Hospital, Iksan, Jeonbuk, Korea. The MRSA strains were defined on the basis of the occurrence of the mecA gene and of their resistance to ampicillin and oxacillin, according to the 2009 guidelines of the Clinical and Laboratory Standards Institute (CLSI) [16]. The standard strains of MRSA ATCC 33591 and S. aureus ATCC 25923, which is a methicillin susceptible Staphylococcus aures (MSSA), were used as control strains. After culturing all stains on Mueller-Hinton agar (MHA), all bacteria were resuspended in Mueller-Hinton broth (MHB) to give $10^{8}$ colony forming units $/ \mathrm{mL}$; the resuspended cells were then incubated.

\subsection{Detection of mecA gene}

Detection of the mecA gene in the MRSA strains was performed by polymerase chain reaction (PCR) amplication. For rapid extraction, one to five bacterial colonies were suspended in $300 \mathrm{~L}$ of cell lysis buffer and heated at $100{ }^{\circ} \mathrm{C}$ for $20 \mathrm{~min}$. After centrifugation at $12,000 \mathrm{rpm}$ for $10 \mathrm{~min}, 2 \mathrm{~L}$ of the supernatant was used for the DNA extraction. PCR reactions were performed using a MRSA Primer Mix Kit (Genotex Co., Korea). The PCR amplification consisted of 30 cycles $\left(94{ }^{\circ} \mathrm{C}, 60\right.$ seconds; $55^{\circ} \mathrm{C}, 60$ seconds; $72{ }^{\circ} \mathrm{C}, 60$ seconds). The primers used in this study were as follows: mecA forward primer, 5'-ATGAGATTAGGCATCGTTTC-3'; reverse primer, 5'-TGGATGACAGTACCTGAGCC-3' [14]. The final PCR products were separated $0.2 \%$ agarose gel. 


\subsection{Minimum Inhibitory Concentration (MIC)}

The standard agar dilution method was used to determine the MIC of the antibiotics alone in accordance with Clinical and Laboratory Standards Institute (CLSI) guidelines [16]. To determine the MIC of A. japonica extract and isolated compounds, the specimen was initially dissolved in minimal amount of dimethyl sulphoxide followed by water to give a range of concentrations from $0.325 \%$ to $5 \%$ with a volume of $2 \mathrm{~mL}$. Each samples were diluted to give a final concentrations from $0.0625 \%$ to $1 \%$ in each agar plate. Cell suspensions $\left(1 \times 10^{4}\right.$ colony-forming units $\left./ \mathrm{mL}\right)$ of $12 \mathrm{MRSA}$ isolates and standard strain (ATCC 25923 and ATCC 33591) were inoculated onto agar plates and then incubated at $35^{\circ} \mathrm{C}$ for $18 \mathrm{~h}$. The MICs were defined as the lowest concentration of a test compound that completely inhibited cell growth in visible.

\section{Acknowledgements}

This paper was supported by Wonkwang University 2009.

\section{References and Notes}

1. Satoh, K.; Nagai, F.; Ushiyama, K.; Kano, I. Specific inhibition of $\mathrm{Na}^{+}, \mathrm{K}(+)$-ATPase activity by atractylon, a major component of byaku-jutsu, by interaction with enzyme in the E2 state. Biochem. Pharmacol. 1996, 51, 339-343.

2. Kitajima, J.; Kamoshita, A.; Ishikawa, T.; Takano, A.; Fukuda, T.; Isoda, S.; Ida, Y. Glycosides of Atractylodes japonica. Chem. Pharm. Bull. 2003, 51, 152-157.

3. Hong, M.H.; Kim, J.H.; Bae, H.; Lee, N.Y.; Shin, Y.C.; Kim, S.H.; Ko, S.G.; Bauer, R. Atractylodes japonica Koidzumi inhibits the production of proinflammatory cytokines through

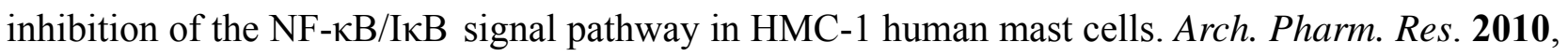
33, 843-851.

4. Yu, K.W.; Kiyohara, H.; Matsumoto, T.; Yang, H.C.; Yamada, H. Intestinal immune system modulating polysaccharides from rhizomes of Atractylodes lancea. Planta Med. 1998, 64, 714-719.

5. Kiso, Y.; Tohkin, M.; Hikino, H. Antihepatotoxic principles of atractylodes rhizomes. J. Nat. Prod. 1983, 46, 651-654.

6. Choi, E.M.; Kim, G.H.; Lee, Y.S. Atractylodes japonica root extract protects osteoblastic MC3T3E1 cells against hydrogen peroxide-induced inhibition of osteoblastic differentiation. Phytother. Res. 2009, 23, 1537-1542.

7. Hikino, H.; Hikino, Y.; Yosioka, I. Studies on the constituents of atractylodes. IX. Structure and autoxidation of atractyon. Chem. Pharm. Bull. 1964, 12, 755-560.

8. Nisikawa, Y.; Watanabe, Y.; Seto, T.; Yasuda, I. Studies on the components of atractylodes. I. New sesquiterpenoids in the rhizone of Atractylodes lancea De Candolle. Yakugaku Zasshi 1976, 96, 1089-1093.

9. Nishikawa, Y.; Seto, T.; Watanabe, Y.; Yasuda, I. Studies on the components of Atractylodes III. New sesquiterpenoid in the rhizome of Atractylodes japonica Koidzumi. Yakugaku Zasshi 1977, 97, 515-518. 
10. Yosioka, I.; Tani, T.; Hirose, M.; Kitagawa, I. Diacetyl-atractylodiol, a new acetylenic compound from Atractylodes japonica Koidzumi. Chem. Pharm. Bull. 1974, 22, 1943-1945.

11. Kano, Y.; Komatsu, K.; Saito, K.; Bando, H.; Sakurai, T. A new polyacetylene compound from atractylodes rhizome. Chem. Pharm. Bull. 1989, 37, 193-194.

12. Lee, S.O.; Seo, J.H.; Lee, J.W.; Yoo, M.Y.; Kwon, J.W.; Choi, S.U.; Kang, J.S.; Kwon, D.Y.; Kim, Y.K.; Kim, Y.S.; Ryu, S.Y. Inhibitory effects of the rhizome extract of Atractylodes japonica on the proliferation of human tumor cell lines. Kor. J. Pharmacogn. 2005, 36, 201-204.

13. Clinical and Laboratory Standards Institute. Methods for dilution antimicrobial susceptibility test for bacteria that grow aerobically; In Approved standard, Document M100-S19, 9th ed.; Clinical and Laboratory Standards Institute: Villanova, PA, USA, 2009.

14. Ryffel, C.W.; Tesch, I.; Birch-Machin, P.E.; Reynolds, L.; Barberis-Maino, F.H.; Kayser, H. Berger-Bächi, B. Sequence comparison of mecA genes isolated from methicillin-resistant Staphylococcus aureus and Staphylococcus epidermidis. Gene 1990, 28, 137-138.

Sample Availability: Samples of the compounds atractylenolide I, atractylenolide III, and diacetylatractylodiol (TDEYA) are available from the authors.

(C) 2010 by the authors; licensee MDPI, Basel, Switzerland. This article is an open access article distributed under the terms and conditions of the Creative Commons Attribution license (http://creativecommons.org/licenses/by/3.0/). 